\title{
Ginkgolide $B$ alleviates the inflammatory response and attenuates the activation of LPS-induced BV2 cells in vitro and in vivo
}

\author{
MIAOXUAN SUN ${ }^{1}$, YI SHENG ${ }^{2}$ and YANYAN ZHU ${ }^{1}$ \\ ${ }^{1}$ Department of Rehabilitation, The First Affiliated Hospital of Wenzhou Medical University; \\ ${ }^{2}$ Department of Anesthesia, Ouhai District Hospital of Integrated Traditional Chinese and \\ Western Medicine, Wenzhou, Zhejiang 325000, P.R. China
}

Received September 18, 2020; Accepted December 23, 2020

DOI: $10.3892 /$ etm.2021.10018

\begin{abstract}
Ginkgolide B (GB) is widely used in the treatment of neurological diseases and exerts anti-inflammatory and neuroprotective effects. Microglia serve an important role in central nervous inflammation. The present study investigated the effect of GB on central nervous inflammation in vivo and in vitro. BV2 cells were activated with lipopolysaccharide (LPS) to establish a cellular model of neuroinflammation. Cell viability was determined using the Cell Counting Kit- 8 assay and the secreted levels of TNF- $\alpha$, IL-1 and IL-6 were measured using ELISAs. The levels of nitric oxide (NO) was assessed using Griess assays. In addition, the mRNA and protein expression levels of inducible NO synthase and cyclooxygenase-2 (COX-2) were detected using reverse transcription-quantitative PCR and western blot analyses, respectively. Transwell assays were carried out to evaluate the cell migratory ability. For the in vivo studies, an LPS-induced neuroinflammation model was established in C57 mice. Western blot analysis and immunohistochemistry were performed to detect the expression of the microglial marker allograft inflammatory factor 1 in the hippocampal dentate gyrus and striatum. The expression levels of TNF- $\alpha$, IL-1 and IL-6 in the hippocampal dentate gyrus and striatum were assessed using western blot analysis. The results revealed that GB reduced the inflammatory response and migration of LPS-induced BV2 cells. Furthermore, GB attenuated the activation of BV2 cells of the hippocampal dentate gyrus and striatum in the LPS-induced mice with neuroinflammation. Taken together, the findings of the present study demonstrated that GB alleviated the inflammatory response and attenuated the activation of LPS-induced BV2 cells in vitro and in vivo.
\end{abstract}

Correspondence to: Dr Miaoxuan Sun, Department of Rehabilitation, The First Affiliated Hospital of Wenzhou Medical University, Park Road, 2 Fuxue Lane, Lucheng, Wenzhou, Zhejiang 325000, P.R. China

E-mail: sunmiaoxuansmx@126.com

Key words: ginkgolide B, neuroinflammation, inflammatory response, microglial cells, BV2 cells

\section{Introduction}

Neuroinflammation is the general termused to describe peripheral nerve inflammation and degenerative diseases caused by various lesions (1). Neuroinflammation is associated with the progression of several neurological diseases and is a sign of neurological complications. In addition, neuroinflammation mainly occurs in the central nervous system (brain) and is usually caused by infection, irritation or trauma (2). Nerve tissue necrosis, cerebral palsy, stroke, brain cancer, brain dementia, brain death and other factors have been associated with neuroinflammation (3).

The central nervous system mainly consists of two types of cells, neurons and glial cells (4). Glial cells are divided into oligodendrocytes, astrocytes and microglial cells (5). Microglial cells are immune effector cells. Central nervous system inflammation is caused by the activation of microglial cells and the release of cytokines and inflammatory mediators (6). Normally, microglial cells are in a resting state as neurons secrete inhibitory factors (7). During injury or disease, under the influence of pro-inflammatory stimuli, microglial cells are activated, secrete cytokines and chemokines, and recruit blood-derived immune cells to the central nervous system, thus amplifying the inflammatory response of the central nervous system (7). However, due to the excessive release of pro-inflammatory cytokines and neurotoxins, M1-type microglial cells may become overactivated and produce excessive amounts of cytokines and chemokines, which in turn, promotes neurotoxicity, damages neurons and can even lead to neuronal death, thus aggravating inflammation of the central nervous system $(8,9)$.

Ginkgolide B (GB) is a terpene lactone in the ginkgolide extracts. Currently, GB is widely used in the treatment of neurological diseases, including cerebral ischemia (10). Emerging evidence has suggested that GB exerts anti-inflammatory and neuroprotective effects $(11,12)$. GB alleviates hypoxia-induced hippocampal neuron injury in mice through inhibiting oxidative stress and cell apoptosis (13). Furthermore, GB promotes the differentiation of neural stem cells following cerebral ischemia-reperfusion injury (14). However, the effect of GB on the inflammatory response and activation of microglial cells, to the best of our knowledge, has not been reported.

The aim of the present study was to investigate the effects of GB on the activation and inflammatory response of microglial cells in vivo and in vitro, thus providing a theoretical basis 
for the clinical application of GB in the treatment of neuroinflammatory diseases.

\section{Materials and methods}

Cell culture and treatment. The murine microglial cell line BV2 was purchased from The Cell Bank of Type Culture Collection of The Chinese Academy of Sciences and cells were cultured in DMEM (Gibco; Thermo Fisher Scientific, Inc.) supplemented with 10\% FBS (Gibco; Thermo Fisher Scientific, Inc.) and penicillin-streptomycin $(100 \mu \mathrm{g} / \mathrm{ml}$; Thermo Fisher Scientific, Inc.) at $37^{\circ} \mathrm{C}$ in a humidified atmosphere with $5 \%$ $\mathrm{CO}_{2}$. The supplemented DMEM was prepared under aseptic conditions (DMEM:FBS, 9:1). GB (cat. no. 15291-77-7; purity, $>98 \%$ ) was purchased from Shanghai Yuanye Biological Technology Co., Ltd. A total of $100 \mathrm{ng} / \mathrm{ml}$ lipopolysaccharide (LPS; cat. no. S1732; Beyotime Institute of Biotechnology) was used to stimulate BV2 in six-well plates at a density of $5 \times 10^{5}$ cells/well for $6 \mathrm{~h}$ at $37^{\circ} \mathrm{C}$ to mimic the inflammatory environment in the brain.

Establishment of neuroinflammation mouse model. A total of 25 male C57BL/6J mice, aged 8 weeks were provided by the First Affiliated Hospital of Wenzhou Medical University (Wenzhou, China). The study protocol was approved by the Ethics Committee of the First Affiliated Hospital of Wenzhou Medical University. All procedures were in compliance with the National Institutes of Health Guide for the Care and Use of Laboratory Animals. To evaluate the anti-inflammatory effects of GB in vivo, mice were divided into the following five groups: Control, LPS, GB $30 \mu \mathrm{mol} / 1+$ LPS, GB $60 \mu \mathrm{mol} / 1+$ LPS and GB $120 \mu \mathrm{mol} / 1+$ LPS groups ( $n=5 /$ group). Mice in the GB + LPS groups were treated daily by gavage with 30,60 and $120 \mu \mathrm{mol} / 1$ of GB for 3 days. Mice in the LPS and control groups were treated with intragastric administration of the same dosage of normal saline for 3 days. Following the last intragastric administration of GB or normal saline for $1 \mathrm{~h}$, the mice in the LPS groups were intraperitoneally injected with $5 \mathrm{mg} / \mathrm{kg}$ of LPS and after $24 \mathrm{~h}$, all mice were anesthetized with intraperitoneal injection of $10 \%$ chloral hydrate $(400 \mathrm{mg} / \mathrm{kg})(15,16)$. The animals treated with chloral hydrate did not exhibit any evident signs of peritonitis. Subsequently, $\sim 0.35 \mathrm{ml}$ of orbital blood was collected from each mouse and the supernatant was isolated by centrifugation at $300 \mathrm{x} \mathrm{g}$ at $4^{\circ} \mathrm{C}$ for $10 \mathrm{~min}$ and stored in a refrigerator at $-80^{\circ} \mathrm{C}$. Mice were then sacrificed by cervical dislocation and euthanasia was confirmed by the cessation of heartbeat. Whole brains were then removed and fixed with $4 \%$ formaldehyde for $24 \mathrm{~h}$ at $4^{\circ} \mathrm{C}$.

Cell Counting Kit-8 (CCK-8) assay. Cell viability was assessed using a CCK- 8 assay (Dojindo Molecular Technologies, Inc.). Cells were seeded in 96-well plates at a density of $1 \times 10^{3}$ cells/well and cultured for $24 \mathrm{~h}$. Subsequently, the cells were treated with LPS and GB as aforementioned. Each well was then supplemented with $10 \mu 1$ of CCK- 8 reagent and the plates were incubated at $37^{\circ} \mathrm{C}$ for $4 \mathrm{~h}$. The absorbance in each well was measured at $450 \mathrm{~nm}$ using a Spectra Max 190 Enzyme standard instrument (Molecular Devices LLC).
ELISA. The secretion levels of IL-6 (cat. no. DY406), IL-1 $\beta$ (cat. no. DY401) and TNF- $\alpha$ (cat. no. DY410) (all from R\&D Systems, Inc.) in plasma and tissues were measured using Duoset ELISA kits according to the manufacturer's instructions.

Reverse transcription-quantitative PCR (RT-qPCR) analysis. Total RNA was extracted from cells and tissues using an RNA Extraction kit (cat. no. 9767) and cDNA was synthesized using the PrimeScript ${ }^{\circledR}$ 1st Strand cDNA Synthesis kit (cat. no. D6110A) (both from Takara Bio, Inc.). The temperature protocol of the reverse transcription was $42^{\circ} \mathrm{C}$ for $30 \mathrm{~min}$, followed by $85^{\circ} \mathrm{C}$ for $5 \mathrm{~min}$, according to the manufacturer's instructions. The thermocycling conditions were as follows: $95^{\circ} \mathrm{C}$ for $10 \mathrm{~min} ; 30$ cycles at $94^{\circ} \mathrm{C}$ for $15 \mathrm{sec}, 55^{\circ} \mathrm{C}$ for $30 \mathrm{sec}$ and $72^{\circ} \mathrm{C}$ for $30 \mathrm{sec}$; and $72^{\circ} \mathrm{C}$ for $10 \mathrm{~min}$, according to the manufacturer's protocol. The PCR reactions were carried out using a SYBR green-based system (cat. no. RR82LR; Takara Bio, Inc.) and the gene fold changes were calculated using the $2^{-\Delta \Delta \mathrm{Cq}}$ method (17). The primer sequences used were as follows: Inducible nitric oxide (NO) synthase (iNOS) forward, 3'-GTCACCTACCAC ACCCGAGATG-5' and reverse, 3'-CGCTGGCATTCCGCA CAA-5'; cyclooxygenase-2 (COX-2) forward, 3'-TGCAGT GAGCGTCAGGAG-5' and reverse, 3'-CAAGGATTTGCT GTATGGCTGAG-5'; and GAPDH (reference gene) forward, 3'-ATCACTGCCACCCAGAAG-5' and reverse, 3'-TCCACG ACGGACACATTG-5'.

Western blot analysis. Total proteins were isolated using a RIPA lysis buffer (Sigma-Aldrich; Merck KGaA) and their concentration was measured using a BCA protein assay kit (Beyotime Institute of Biotechnology). Proteins (25 $\mu \mathrm{g} /$ lane) were separated by $10 \%$ SDS-polyacrylamide gel electrophoresis and transferred onto PVDF membranes. Membranes were blocked with 5\% skimmed milk (Beyotime Institute of Biotechnology) for $1 \mathrm{~h}$ at room temperature. Following blocking, the membranes were first incubated with primary antibodies overnight at $4^{\circ} \mathrm{C}$ and then with the corresponding goat anti-rabbit horseradish peroxidase-conjugated secondary antibodies (dilution, 1:5,000; cat. no. ab181658; Abcam) at room temperature for $2 \mathrm{~h}$. Finally, The ECL ${ }^{\mathrm{TM}}$ Western Blotting Analysis System (Cytiva) and the ImageJ software (version 1.46; National Institutes of Health) were used to detect the protein blots. In the present study, the following primary antibodies were used: Anti-iNOS (dilution, 1:1,000; cat. no. ab178945), anti-COX-2 (dilution, 1:1,000; cat. no. ab179800), anti-allograft inflammatory factor 1 (Iba-1; dilution, 1:1,000; cat. no. ab178846), anti-TNF- $\alpha$ (dilution, 1:1,000; cat. no. ab215188), anti-IL-1 $\beta$ (dilution, 1:1,000; cat. no. ab2105), anti-IL-6 (dilution, 1:1,000; cat. no. ab233706) and anti-GAPDH (dilution, 1:1,000; cat. no. ab8245) (all from Abcam). An additional anti-GAPDH antibody (dilution, 1:1,000; cat. no. 5174S) was obtained from Cell Signaling Technology, Inc.

Determination of $\mathrm{NO}$ production. NO levels in the culture medium were directly measured using Total Nitric Oxide Assay Kit (cat. no. S0023; Beyotime Institute of Biotechnology). Culture supernatants $(50 \mu \mathrm{l})$ were mixed with $100 \mu \mathrm{l}$ Griess reagent and incubated for $3 \mathrm{~min}$ at room temperature. The 
absorbance of each reaction was measured at $540 \mathrm{~nm}$ on a microplate spectrophotometer.

Transwell assay. A Transwell chamber (24-well; 8.0- $\mu \mathrm{m}$ pore membranes; Corning, Inc.) was used according to the manufacturer's instructions. Briefly, the inserts had been precoated with Matrigel (BD Biosciences) at $37^{\circ} \mathrm{C}$ for $30 \mathrm{~min}$. $10^{5}$ cells/well were seeded into the upper chamber in $100 \mu 1$ of serum-free DMEM, while the lower chamber was supplemented with $600 \mu \mathrm{l}$ of DMEM supplemented with $10 \%$ FBS as a chemoattractant for $24 \mathrm{~h}$ at $37^{\circ} \mathrm{C}$. Cells on the upper surface of the membrane were removed using cotton swabs, whereas cells on the lower chamber were fixed with $4 \%$ paraformaldehyde for $20 \mathrm{~min}$ at room temperature and stained with $0.1 \%$ crystal violet solution for $30 \mathrm{~min}$ at room temperature. Finally, the cells were counted under a light contrast microscope (Olympus Corporation; magnification, x200).

Immunohistochemistry. Tissue samples were fixed in formalin for $24 \mathrm{~h}$ at room temperature, embedded in paraffin, sectioned in $4-5 \mu \mathrm{m}$ thickness, and analyzed by immunohistochemistry. Briefly, the samples were blocked with $5 \%$ normal goat serum (cat. no. A7007; Beyotime Institute of Biotechnology) for $1 \mathrm{~h}$ at room temperature, probed with anti-Iba-1 (dilution, 1:1,000; cat. no. ab178846; Abcam) at $4^{\circ} \mathrm{C}$ overnight and labeled with an anti-rabbit HRP secondary antibody (cat. no. ab6721; Abcam) for $1 \mathrm{~h}$ at room temperature. Antibody reactions were visualized using 3,3'-diaminobenzidine chromogen staining for $15 \mathrm{~min}$ at room temperature and the slides were then counterstained with hematoxylin for $3 \mathrm{~min}$ at room temperature. Stained tissue sections were observed under a light microscope (Leica Microsystems, Inc.; magnification, x200).

Statistical analysis. All data were analyzed with the GraphPad Prism 7.0 software (GraphPad Software, Inc.). Comparisons among multiple groups were analyzed using one-way ANOVA followed by Tukey's post hoc test. The data in the present study are presented as the mean \pm SD. All experiments were repeated three times independently. $\mathrm{P}<0.05$ was considered to indicate a statistically significant difference.

\section{Results}

$G B$ reduces the inflammatory response and oxidative stress of LPS-induced BV2 cells. A CCK-8 assay was used to evaluate the effect of GB at various concentrations $(0,30,60$ and $120 \mu \mathrm{mol} / \mathrm{l})$ on the activity of LPS-induced BV2 cells. The results are presented in Fig. 1. There was no significant difference in the viability of cells treated with or without LPS, indicating that treatment of cells with LPS and GB at the various concentrations had no significant effect on the activity of BV2 cells. Therefore, cells were first induced with LPS to establish the BV2 cell model and then treated with different concentrations of GB. ELISAs were carried out to determine the effects of GB on LPS-induced inflammatory factors and oxidative stress. The results demonstrated that the LPS-induced expression levels of IL- $1 \beta$, IL- 6 and TNF- $\alpha$ were significantly increased compared with that in the control group. The secretion levels of IL-1 $\beta$, IL- 6 and TNF- $\alpha$ in the GB + LPS group were notably decreased in a dose-dependent

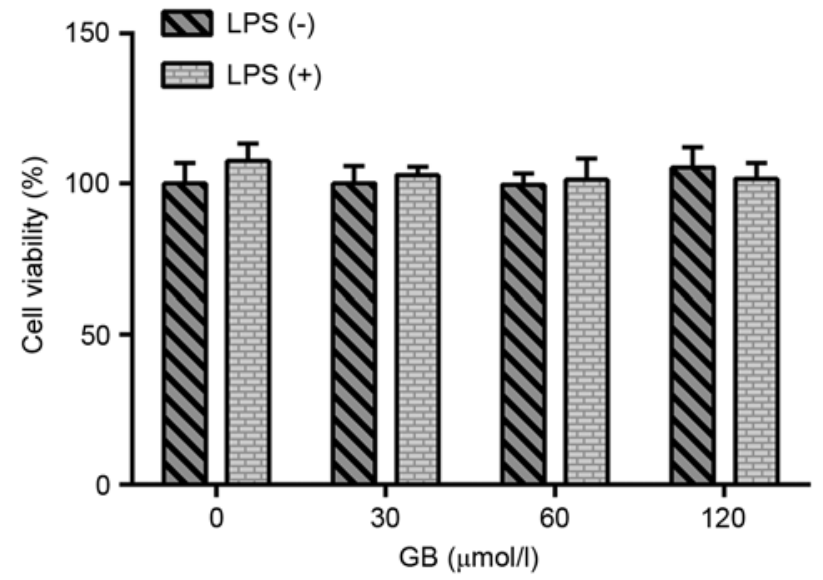

Figure 1. Effects of GB on cell viability. Cell Counting Kit-8 assays were used to detect the viability of LPS-treated cells. GB, ginkgolide B; LPS, lipopolysaccharide.

manner compared with the LPS group (Fig. 2A). Furthermore, Griess assays were used to measure the levels of NO. Compared with the control group, the levels of NO were significantly increased in the LPS group. Additionally, the expression levels of NO in the GB + LPS groups were reduced in a concentration-dependent manner compared with the LPS group (Fig. 2B). The expression patterns of iNOS and COX-2 were consistent with that of NO, as determined by western blotting (Fig. 2C) and RT-qPCR (Fig. 2D) analyses. These findings indicated that GB attenuated the LPS-induced inflammatory response and oxidative stress.

$G B$ alleviates the LPS-induced migration of BV2 cells. A transwell assay was carried out to evaluate the cell migratory ability. As shown in Fig. 3A and B, compared with the untreated control group, the cell migratory ability in the LPS group was notably enhanced. In addition, the cell migratory ability in the GB + LPS groups was decreased in a dose-dependent manner compared with the LPS group. These results suggested that GB alleviated LPS-induced BV2 cell migration.

$G B$ reduces the activation of microglial cells in the hippocampal dentate gyrus and striatum of LPS-induced mice. Subsequently, the in vivo effects of GB on the activation of BV2 cells and the inflammatory response of cells of the hippocampal dentate gyrus and striatum microglia were investigated in LPS-induced mice. The expression levels of Iba-1, a microglial cell surface protein marker, were determined to evaluate the activation status of BV2 (18). The western blotting results revealed that, compared with the control group, the expression levels of Iba-1 in the dentate gyrus of the hippocampus of mice were significantly increased. In addition, compared with the LPS group, Iba-1 expression in the GB + LPS group was notably decreased in a GB concentration-dependent manner (Fig. 4A). The expression status of Iba-1 in the striatum was consistent with that in the dentate gyrus (Fig. 4B). In addition, the expression of Iba-1 in the hippocampal dentate gyrus (Fig. 4C) and striatum (Fig. 4D) of mice was also evaluated by immunohistochemistry. Compared with the control group, 
A
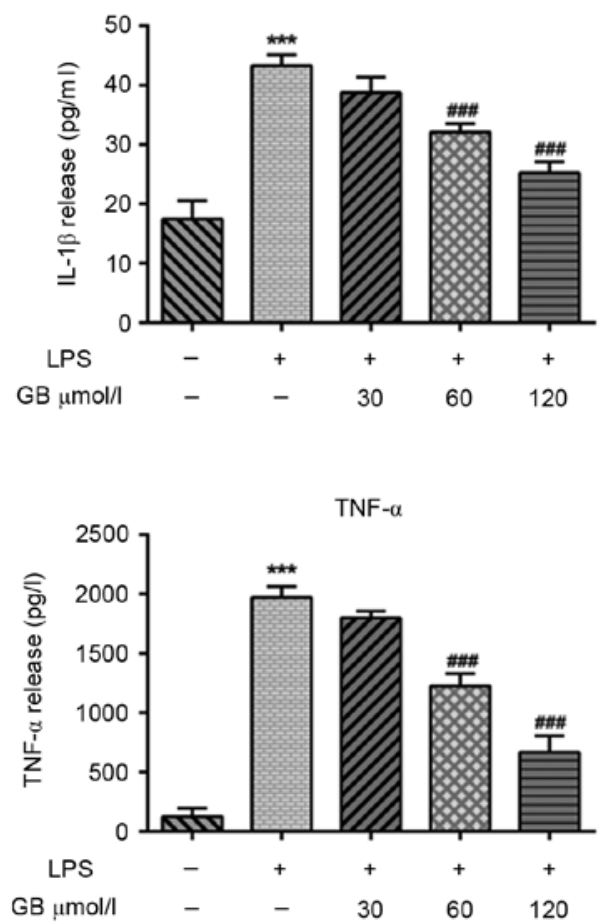

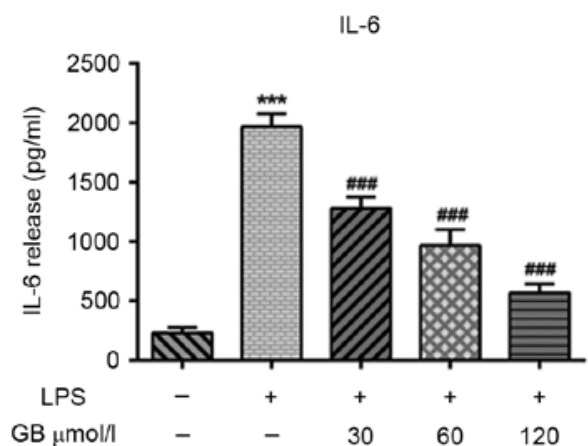

B

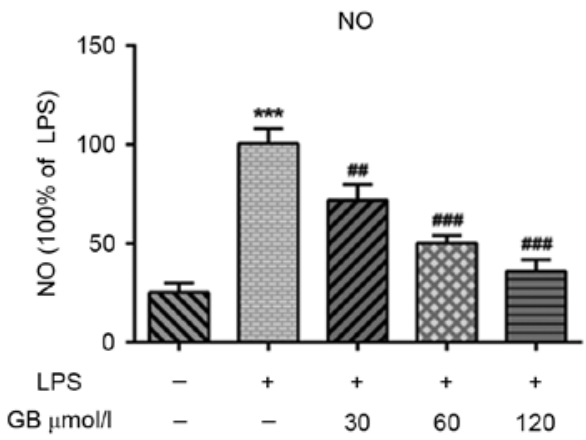

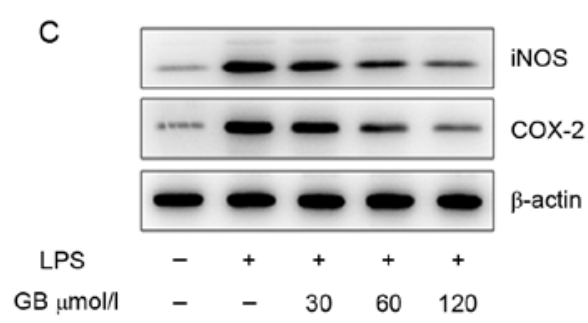
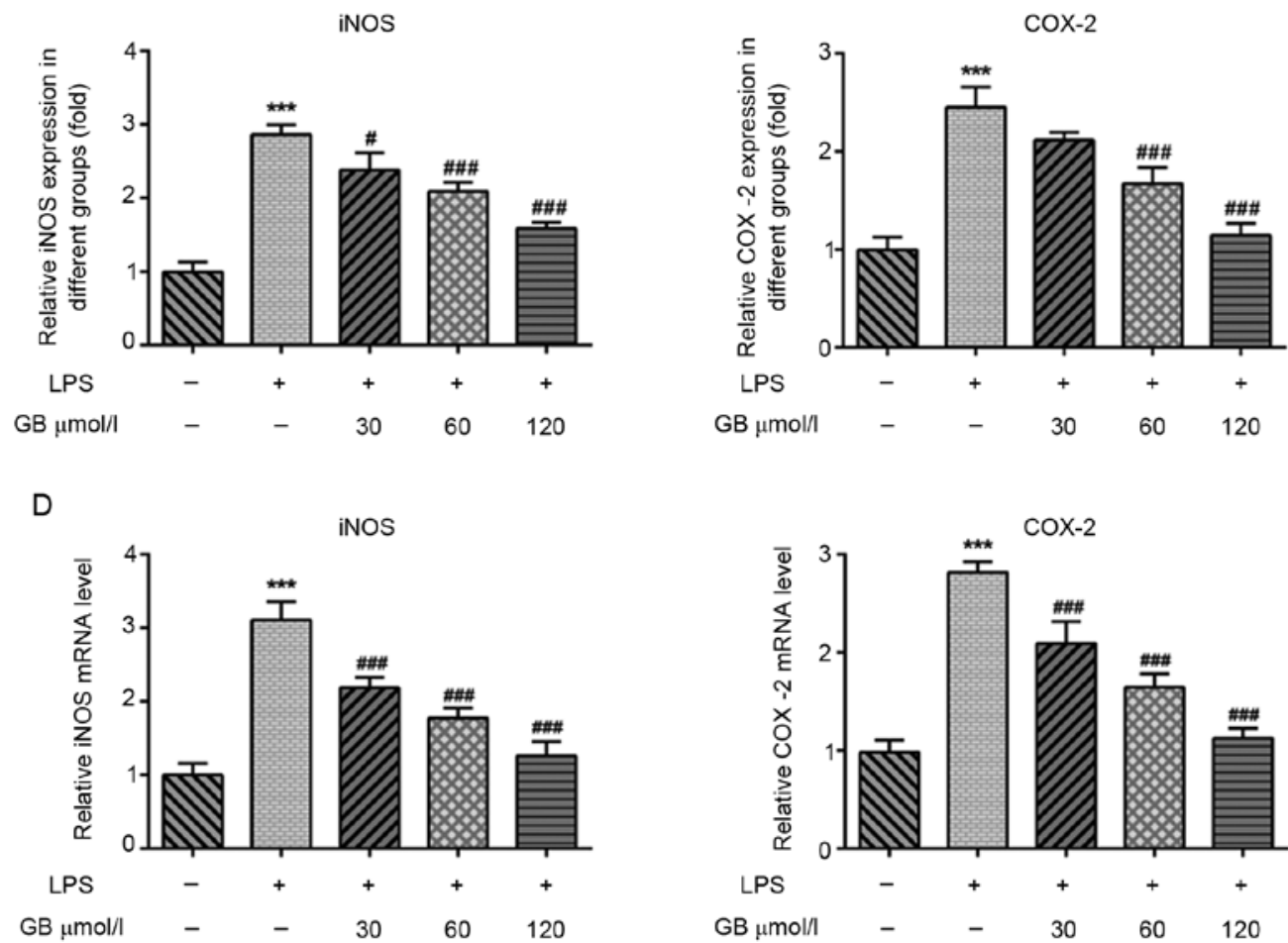

Figure 2. GB reduces the inflammatory response and oxidative stress of BV2 cells induced by LPS. (A) The expression levels of inflammatory cytokines in cells were detected using ELISA. (B) Griess assays detected the expression of NO. (C) Western blotting detected the protein expression levels of oxidative stress-related proteins. (D) reverse transcription-quantitative PCR detected the mRNA expression levels of oxidative stress related proteins. ${ }^{* * *} \mathrm{P}<0.001$ vs. control; ${ }^{\# \mathrm{P}}<0.05,{ }^{\# \#} \mathrm{P}<0.01$ and ${ }^{\# \#} \mathrm{P}<0.001$ vs. the LPS only-treated group. COX-2, cyclooxygenase-2; GB, ginkgolide B; iNOS, inducible NO synthase; LPS, lipopolysaccharide; NO, nitric oxide. 

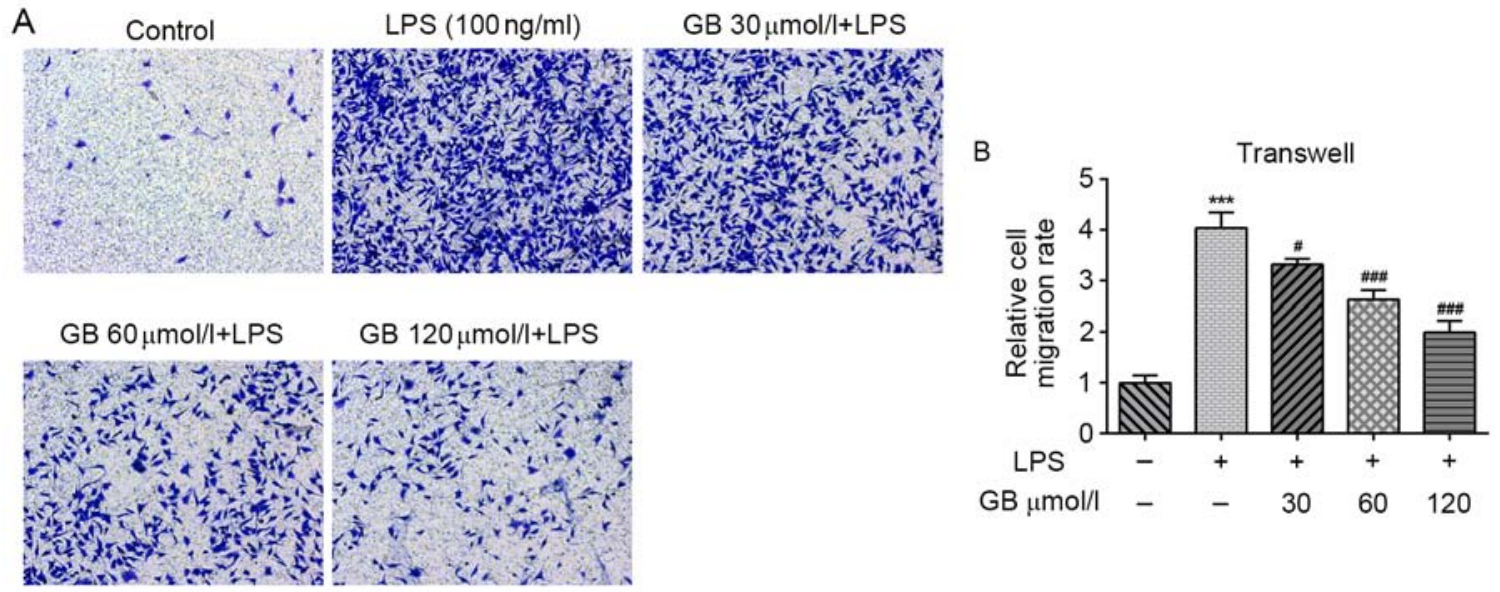

Figure 3. GB alleviates migration of BV2 cells induced by LPS. (A) Transwell assays were used to detect the cell invasion rate (magnification, x200). (B) Quantification of the cell invasion rate. ${ }^{* * * *} \mathrm{P}<0.001$ vs. control; ${ }^{\#} \mathrm{P}<0.05$ and ${ }^{\# \# \#} \mathrm{P}<0.001$ vs. the LPS only-treated group. GB, ginkgolide $\mathrm{B}$; LPS, lipopolysaccharide.

A
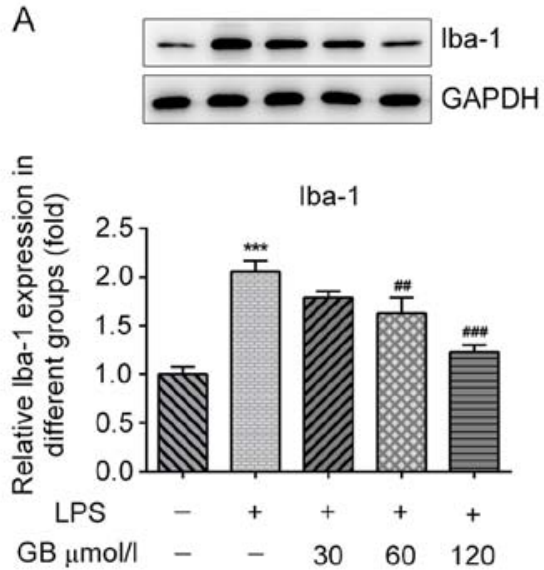

B
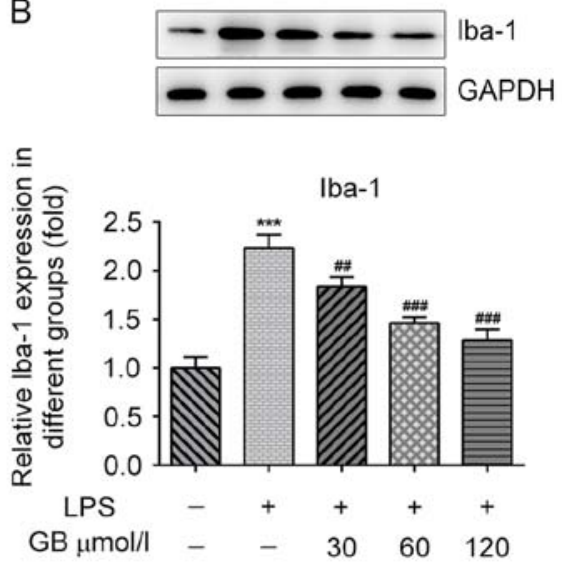

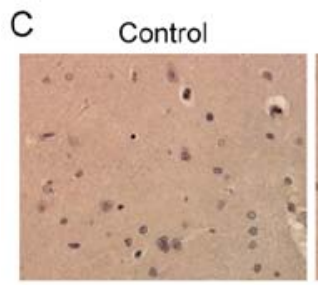

\section{PLS (100 ng/ml)}
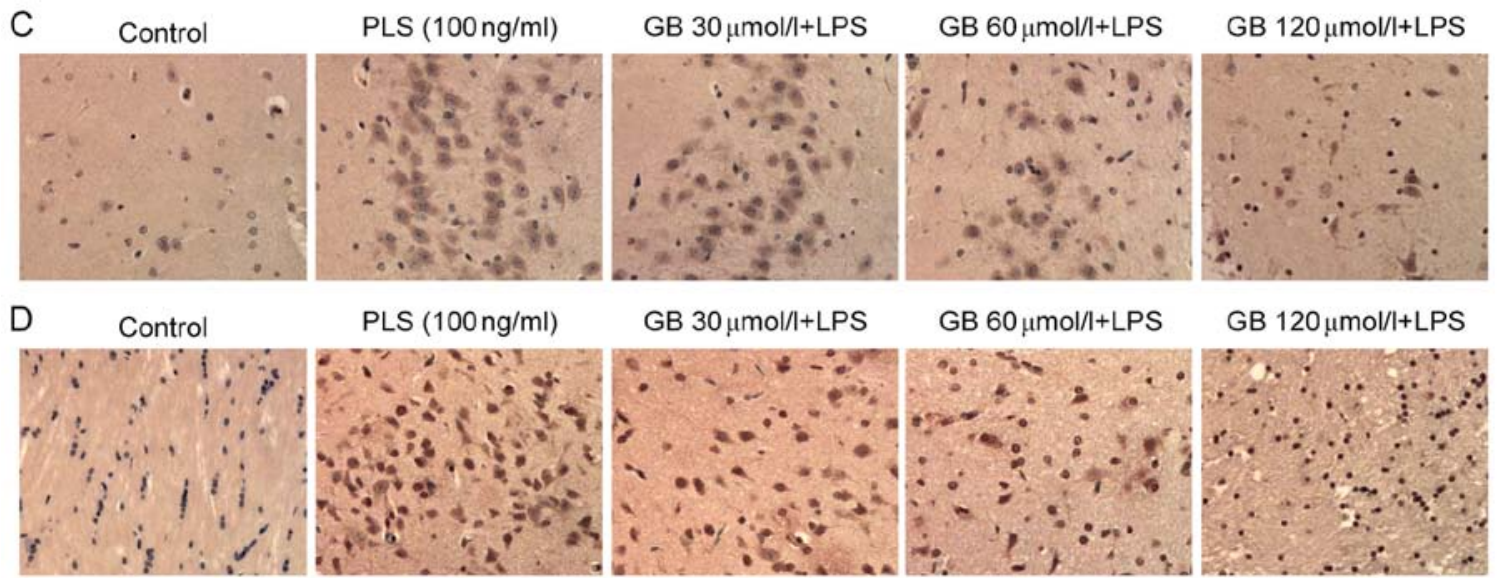

GB $60 \mu \mathrm{mol} / \mathrm{l}+\mathrm{LPS}$

GB $120 \mu \mathrm{mol} / \mathrm{l}+\mathrm{LPS}$
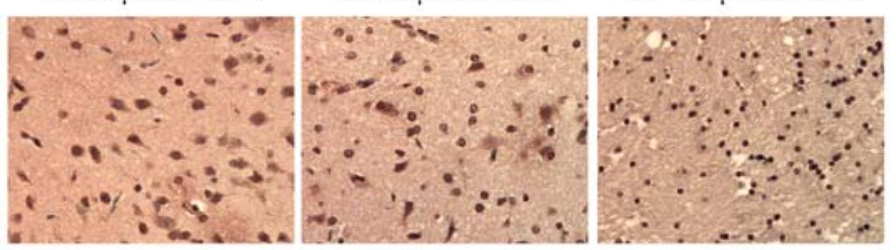

Figure 4. GB attenuates the activation of microglial cells in hippocampal dentate gyrus and striatum of mice induced by LPS. Western blotting detected the protein expression levels of Iba-1 in the (A) hippocampal dentate gyrus and (B) striatum. Immunohistochemistry detected the expression of Iba-1 in the (C) hippocampal dentate gyrus and (D) striatum. Magnification, x 200. ${ }^{* * *} \mathrm{P}<0.001$ vs. control; ${ }^{\# \#} \mathrm{P}<0.01$ and ${ }^{\# \#} \mathrm{P}<0.001$ vs. the LPS only-treated group. GB, ginkgolide B; Iba-1, allograft inflammatory factor 1; LPS, lipopolysaccharide.

more Iba-1 positive cells in the dentate gyrus of the hippocampus and striatum were apparent in the LPS group. In addition, compared with the LPS group, the number of Iba-1 positive cells in the dentate gyrus of the hippocampus and striatum in the GB + LPS group was notably decreased in a GB concentration-dependent manner. The results were consistent with those observed in the western blot analysis. Overall, the results demonstrated that GB attenuated the activation of LPS-induced microglial cells in the hippocampal dentate gyrus and striatum. 
A
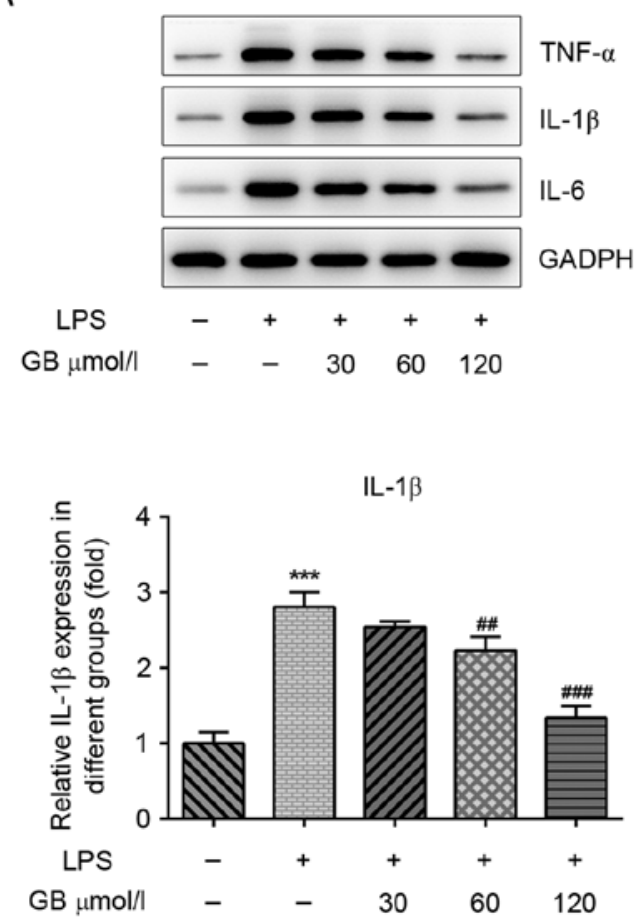

B
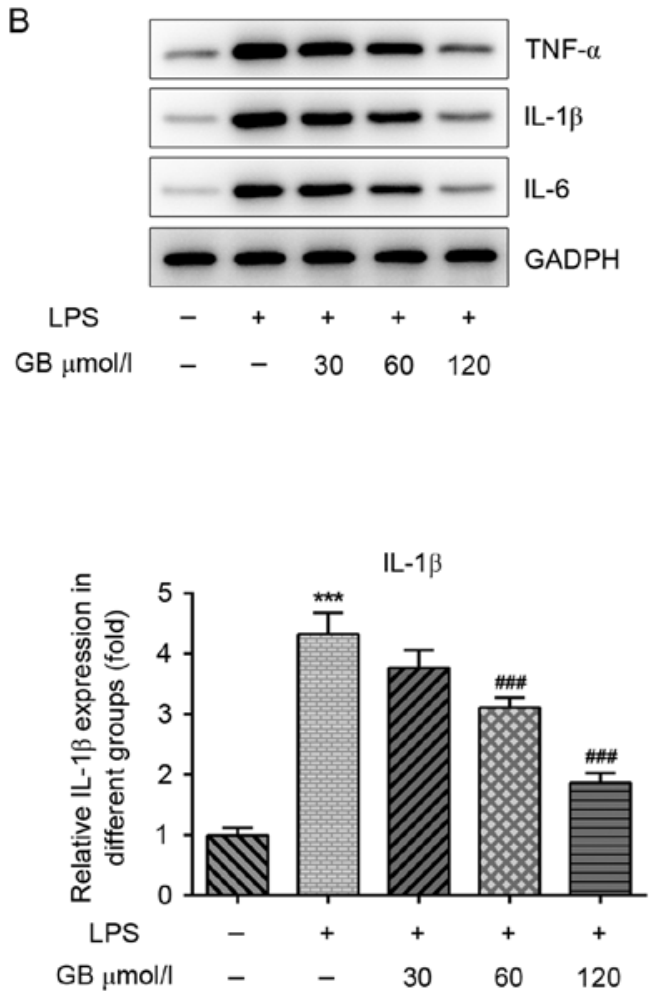
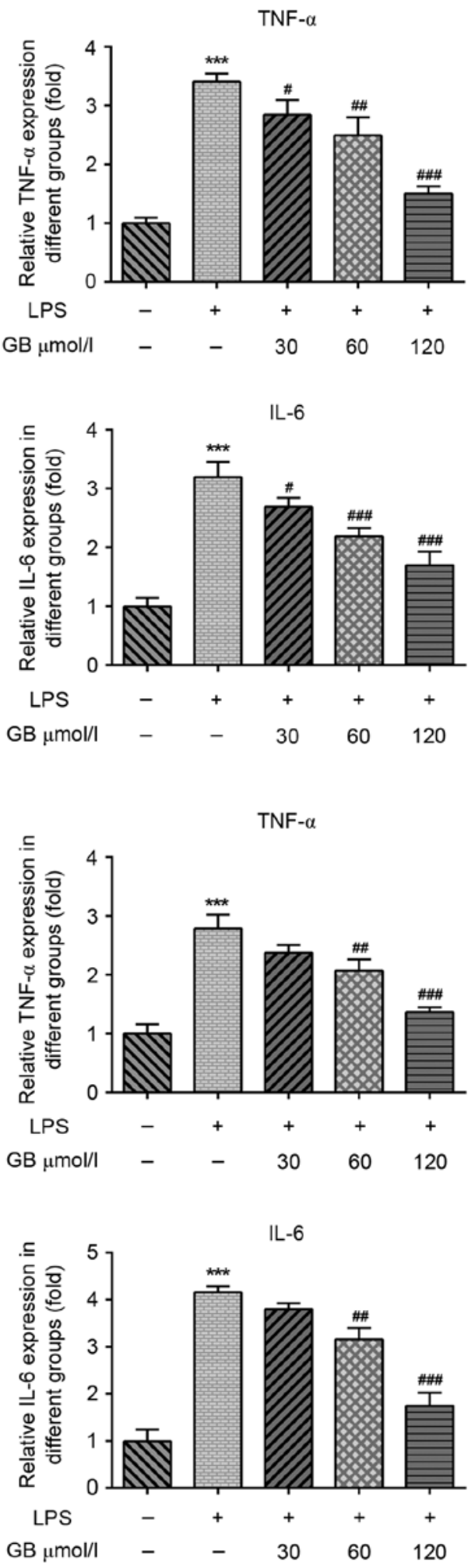

Figure 5. GB reduces the inflammatory response in the hippocampal dentate gyrus and striatum of mice induced by LPS. The protein expression levels of inflammatory cytokines in cells were detected using western blotting in the (A) hippocampal dentate gyrus and (B) striatum. ${ }^{* * *} \mathrm{P}<0.001 \mathrm{vs}$. control; ${ }^{\#} \mathrm{P}<0.05$, ${ }^{\# \#} \mathrm{P}<0.01$ and ${ }^{\# \#} \mathrm{P}<0.001$ vs. the LPS only-treated group. GB, ginkgolide $\mathrm{B} ; \mathrm{LPS}$, lipopolysaccharide.

GB reduces the inflammatory response in the hippocampal dentate gyrus and striatum of LPS-induced mice. Subsequently, the expression levels of TNF- $\alpha$, IL-1 $\beta$ and IL- 6 in the hippocampal dentate gyrus and striatum of LPS-induced mice were also detected, (Fig. 5). The western blotting results revealed that the protein expression levels of TNF- $\alpha$, IL- $1 \beta$ and IL- 6 in the hippocampal dentate gyrus of LPS-induced mice were significantly increased compared with that in the control group. Compared with the LPS only-treated group, the expression of TNF- $\alpha$, IL- $1 \beta$ and IL- 6 in the hippocampal dentate gyrus of mice treated with various concentrations of GB + LPS was decreased in a dose-dependent manner (Fig. 5A). The 
expression patterns of all three cytokines in the striatum were consistent with those observed in the hippocampal dentate gyrus (Fig. 5B). These findings indicated that GB reduced the expression of TNF- $\alpha$, IL- $1 \beta$ and IL- 6 in the hippocampal dentate gyrus and striatum of LPS-induced mice.

\section{Discussion}

In the present study, BV2 microglial cells were induced with LPS to establish a neuroinflammation cell model in vitro. Various concentrations of GB inhibited the inflammatory response and cell activation of LPS-induced BV2 microglial cells. In vivo, the results demonstrated that GB attenuated the activation and inflammatory response of microglial cells in the hippocampal dentate gyrus and striatum in the neuroinflammation mouse model.

Neuroinflammation is a complex and ordered process, involving a variety of glial and peripheral immune cells of the central nervous system (7). Neuroinflammation is usually mediated by activated microglia and astrocytes via the secretion of pro-inflammatory cytokines (19). Microglia are the resident immune cells in the brain (20). A moderate neuroinflammatory response mediated by microglia is the defense mechanism of the central nervous system against infection and injury (21), while persistent or excessive inflammatory responses can lead to progressive nerve cell damage and dysfunction (22). A previous study has shown that excessive neuroinflammation induced by microglia activation is an important pathological mechanism for the occurrence and development of neurodegenerative diseases (23). Therefore, inhibiting the continuous activation of microglia and reducing the excessive release of pro-inflammatory mediators are considered to be effective interventions for the prevention and treatment of neurodegenerative diseases (24). Herein, BV2 microglial cells were induced with LPS to establish a neuroinflammation model. Subsequently, the activation of hippocampal dentate gyrus and striatum microglia was evaluated following LPS induction in mice to determine whether the neuroinflammatory response was successfully induced.

$\mathrm{NO}$ is an important inflammatory mediator produced by the body and its excessive production is closely associated with various neurodegenerative diseases (25). Microglia express iNOS in the activated state, which promotes the production of a large amount of NO (16). Excessive NO activates NF- $\kappa \mathrm{B}$ and induces the production of inflammatory factors such as IL-1 $\beta$ and TNF- $\alpha$. The production of these inflammatory factors, in turn, activates iNOS and further promotes the body to produce more NO, thereby producing a sustained toxic effect on cells. It has been reported that tissue trauma or infection may cause the release of pro-inflammatory cytokines, such as peripheral and central IL-1 $\beta$, TNF- $\alpha$ and IL-6 (26). A previous study showed that Porphyromonas gingivalis LPS induced neuronal inflammation in C57BL/6 mice and significantly increased the expression levels of IL-6, TNF- $\alpha$ and IL-1 $\beta$ (27). In the present study, the expression of TNF- $\alpha$, IL-1 $\beta$ and IL- 6 in BV2 cells, as well as the hippocampal dentate gyrus and striatum of C57BL/6 mice, was also significantly increased following induction with LPS.

GB exerts anti-inflammatory and antioxidant pharmacological effects (28). In HUVECs, GB mediates the inhibition of inflammatory cascades and alters lipid metabolism through targeting the expression and function of proprotein convertase subtilisin/kexin type 9 (11). In addition, GB regulates myocardial inflammation induced by ischemia-reperfusion injury via the A20/NF- $\mathrm{B}$ signaling pathway (29). In the present study, GB inhibited the expression of inflammation-related factors in LPS-induced microglial cells and inhibited the activation of microglial cells, suggesting that GB may serve a therapeutic role in LPS-induced neuroinflammation.

Attention should be paid to the following limitations in the present study. Through in vivo and in vitro experiments, it was demonstrated that GB inhibited LPS-induced neuroinflammation and microglial cell activation; however, the specific mechanism of action remains to be further explored. In addition, the effect of GB on other cells in neuroinflammation, such as neuronal cells, oligodendrocytes or astrocytes, and whether GB can also inhibit the inflammation and activation of these cells remains to be explored.

Overall, the findings of the present study revealed that GB alleviated the inflammatory response and activation of LPS-induced BV2 cells in vivo and in vitro, thus providing the theoretical basis for the clinical application of GB in the treatment of neuroinflammation.

\section{Acknowledgements}

Not applicable.

\section{Funding}

No funding was received.

\section{Availability of data and materials}

The datasets used and/or analyzed during the current study are available from the corresponding author on reasonable request.

\section{Authors' contributions}

MS wrote the manuscript and analyzed the data. YS and YZ carried out the experiments, supervised the present study, searched the literature and revised the manuscript. MS and YS confirm the authenticity of all the raw data. All authors have read and approved the final manuscript.

\section{Ethics approval and consent to participate}

The study protocol was approved by Ethics Committee of the First Affiliated Hospital of Wenzhou Medical University (Wenzhou, China). All the procedures were in compliance with The National Institutes of Health Guide for the Care and Use of Laboratory Animals.

\section{Patient consent for publication}

Not applicable.

\section{Competing interests}

The authors declare that they have no competing interests. 


\section{References}

1. Klein RS, Garber C and Howard N: Infectious immunity in the central nervous system and brain function. Nat Immunol 18: 132-141, 2017.

2. Schain $M$ and Kreisl WC: Neuroinflammation in neurodegenerative disorders-a review. Curr Neurol Neurosci Rep 17: 25, 2017.

3. Matsuda M, Huh Y and Ji RR: Roles of inflammation, neurogenic inflammation, and neuroinflammation in pain. J Anesth 33: 131-139, 2019.

4. Song I and Dityatev A: Crosstalk between glia, extracellular matrix and neurons. Brain Res Bull 136: 101-108, 2018.

5. Allen NJ and Lyons DA: Glia as architects of central nervous system formation and function. Science 362: 181-185, 2018.

6. Kinney JW, Bemiller SM, Murtishaw AS, Leisgang AM, Salazar AM and Lamb BT: Inflammation as a central mechanism in Alzheimer's disease. Alzheimers Dement (N Y) 4: 575-590, 2018.

7. Yang QQ and Zhou JW: Neuroinflammation in the central nervous system: Symphony of glial cells. Glia 67: 1017-1035, 2019.

8. Gonzalez H, Elgueta D, Montoya A and Pacheco R: Neuroimmune regulation of microglial activity involved in neuroinflammation and neurodegenerative diseases. J Neuroimmunol 274: 1-13, 2014

9. Burguillos MA, Deierborg T, Kavanagh E, Persson A, Hajji N, Garcia-Quintanilla A, Cano J, Brundin P, Englund E, Venero JL and Joseph B: Caspase signalling controls microglia activation and neurotoxicity. Nature 472: 319-324, 2011.

10. Nabavi SM, Habtemariam S, Daglia M, Braidy N, Loizzo MR, Tundis R and Nabavi SF: Neuroprotective effects of ginkgolide B against ischemic stroke: A review of current literature. Curr Top Med Chem 15: 2222-2232, 2015.

11. Wang G, Liu Z, Li M, Li Y, Alvi SS, Ansari IA and Khan MS: Ginkgolide B mediated alleviation of inflammatory cascades and altered lipid metabolism in HUVECs via targeting PCSK-9 expression and functionality. Biomed Res Int 2019 7284767, 2019.

12. Feng Z, Sun Q, Chen W, Bai Y, Hu D and Xie X: The neuroprotective mechanisms of ginkgolides and bilobalide in cerebral ischemic injury: A literature review. Mol Med 25: 57, 2019.

13. Zheng PD, Mungur R, Zhou HJ, Hassan M, Jiang SN and Zheng JS: Ginkgolide B promotes the proliferation and differentiation of neural stem cells following cerebral ischemia/ reperfusion injury, both in vivo and in vitro. Neural Regen Res 13: 1204-1211, 2018.

14. Li MY, Chang CT, Han YT, Liao CP, Yu JY and Wang TW: Ginkgolide B promotes neuronal differentiation through the $\mathrm{Wnt} / \beta$-catenin pathway in neural stem cells of the postnatal mammalian subventricular zone. Sci Rep 8: 14947, 2018.

15. Li W, Qinghai S, Kai L, Xue M, Lili N, Jihua R, Zhengxiang L, Xiaoling L, Di G, Qi Y, et al: Oral administration of Ginkgolide B alleviates hypoxia-induced neuronal damage in rat hippocampus by inhibiting oxidative stress and apoptosis. Iran J Basic Med Sci 22: 140-145, 2019.

16. Qu Z, Chen Y, Luo ZH, Shen XL and Hu YJ: 7-methoxyflavanone alleviates neuroinflammation in lipopolysaccharide-stimulated microglial cells by inhibiting TLR4/MyD88/MAPK signalling and activating the Nrf2/NQO-1 pathway. J Pharm Pharmacol 72 : $385-395,2020$
17. Livak KJ and Schmittgen TD: Analysis of relative gene expression data using real-time quantitative PCR and the 2(-Delta Delta C(T)) method. Methods 25: 402-408, 2001.

18. Norden DM, Trojanowski PJ, Villanueva E, Navarro E and Godbout JP: Sequential activation of microglia and astrocyte cytokine expression precedes increased Iba-1 or GFAP immunoreactivity following systemic immune challenge. Glia 64 : 300-316, 2016.

19. Shabab T, Khanabdali R, Moghadamtousi SZ, Kadir HA and Mohan G: Neuroinflammation pathways: A general review. Int J Neurosci 127: 624-633, 2017.

20. Ottum PA, Arellano G, Reyes LI, Iruretagoyena M and Naves R: Opposing roles of interferon-gamma on cells of the central nervous system in autoimmune neuroinflammation. Front Immunol 6: 539, 2015.

21. Glass CK, Saijo K, Winner B, Marchetto MC and Gage FH: Mechanisms underlying inflammation in neurodegeneration. Cell 140: 918-934, 2010.

22. Ferreira ST, Clarke JR, Bomfim TR and De Felice FG: Inflammation, defective insulin signaling, and neuronal dysfunction in Alzheimer's disease. Alzheimers Dement 10 (1 Suppl): S76-S83, 2014.

23. Subhramanyam CS, Wang $\mathrm{C}, \mathrm{Hu}$ Q and Dheen ST: Microglia-mediated neuroinflammation in neurodegenerative diseases. Semin Cell Dev Biol 94: 112-120, 2019.

24. Tang Y and Le W: Differential roles of M1 and M2 microglia in neurodegenerative diseases. Mol Neurobiol 53: 1181-1194, 2016.

25. Baruch K, Kertser A, Porat Z and Schwartz M: Cerebral nitric oxide represses choroid plexus $\mathrm{NF \kappa B}$-dependent gateway activity for leukocyte trafficking. EMBO J 34: 1816-1828, 2015.

26. Han PF, Wei L, Duan ZQ, Zhang ZL, Chen TY, Lu JG, Zhao RP, Cao XM, Li PC, Lv Z and Wei XC: Contribution of IL-1 $\beta, 6$ and TNF- $\alpha$ to the form of post-traumatic osteoarthritis induced by 'idealized' anterior cruciate ligament reconstruction in a porcine model. Int Immunopharmacol 65: 212-220, 2018.

27. Zhang J, Yu C, Zhang X, Chen H, Dong J, Lu W, Song Z and Zhou W: Porphyromonas gingivalis lipopolysaccharide induces cognitive dysfunction, mediated by neuronal inflammation via activation of the TLR4 signaling pathway in C57BL/6 mice. J Neuroinflammation 15: 37, 2018.

28. Zhu PC, Tong Q, Zhuang Z, Wang ZH, Deng LH, Zheng GQ and Wang Y: Ginkgolide B for myocardial ischemia/ reperfusion injury: A preclinical systematic review and meta-analysis. Front Physiol 10: 1292, 2019.

29. Zhang R, Xu L, Zhang D, Hu B, Luo Q, Han D, Li J and Shen C: Cardioprotection of ginkgolide B on myocardial ischemia/reperfusion-induced inflammatory injury via regulation of A20-NF- $\kappa$ B pathway. Front Immunol 9: 2844, 2018.

This work is licensed under a Creative Commons Attribution-NonCommercial-NoDerivatives 4.0 International (CC BY-NC-ND 4.0) License. 